\title{
Correction: Summary of Glaucoma Diagnostic Testing Accuracy: An Evidence-Based Meta-Analysis
}

\author{
Saad Ahmed ${ }^{\mathrm{a}}$, Zainab Khan ${ }^{\mathrm{b}}$, Francie $\mathrm{Si}^{\mathrm{a}}$, Alex Mao ${ }^{\mathrm{a}}$, Irene Pan ${ }^{\mathrm{a}}$, Fatemeh Yazdic, \\ Alexander Tsertsvadze ${ }^{\mathrm{c}}$, Cindy Hutnik ${ }^{\mathrm{a}}$, David Moherc ${ }^{\mathrm{c}}$, David Tingey ${ }^{\mathrm{a}}$, Graham E. Trope ${ }^{\mathrm{d}}$, \\ Karim F. Damjie, Jean-Eric Tarride ${ }^{\mathrm{f}}$, Ron Goeree ${ }^{\mathrm{f}}$, Omar Akhtarg, William Hodge ${ }^{\mathrm{a}, \mathrm{g}, \mathrm{h}}$
}

Corrections to article "Summary of Glaucoma Diagnostic Testing Accuracy: An Evidence-Based Meta-Analysis", by Saad Ahmed et al, published in Vol. 8, No. 9, 2016, p641-649, doi: http://dx.doi.org/10.14740/jocmr2643w. The authors of this article would like to add an author, Omar Akhtar, who is entitled to authorship, the new author's list should read as follows.

Saad Ahmed ${ }^{\mathrm{a}}$, Zainab Khan ${ }^{\mathrm{b}}$, Francie $\mathrm{Si}^{\mathrm{a}}$, Alex Mao ${ }^{\mathrm{a}}$, Irene Pan ${ }^{\mathrm{a}}$, Fatemeh Yazdic ${ }^{\mathrm{c}}$, Alexander Tsertsvadze ${ }^{\mathrm{c}}$, Cindy Hutnik ${ }^{\mathrm{a}}$, David Moher $^{\mathrm{c}}$, David Tingey ${ }^{\mathrm{a}}$, Graham E. Trope ${ }^{\mathrm{d}}$, Karim F. Damjie , Jean-Eric Tarridef ${ }^{\mathrm{f}}$, Ron Goeree ${ }^{\mathrm{f}}$, Omar Akhtar', William Hodge ${ }^{\mathrm{a}, \mathrm{g}, \mathrm{h}}$

avey Eye Institute, University of Western Ontario, London, ON, Canada

${ }^{b}$ Faculty of Medicine, Queen's University, Kingston, ON, Canada

'Ottawa Hospital Research Institute, University of Ottawa, Ottawa, ON, Canada

${ }^{\mathrm{d} D e p a r t m e n t ~ o f ~ O p h t h a l m o l o g y ~ a n d ~ V i s u a l ~ S c i e n c e s, ~ U n i v e r s i t y ~ o f ~ T o r o n t o, ~ T o r o n t o, ~ O N, ~ C a n a d a ~}$

eDepartment of Ophthalmology, University of Alberta, Edmonton, AB, Canada

fProgram for the Assessment of Technology and Health (PATH), and Department of Clinical Epidemiology and Biostatistics, McMaster University, Hamilton, ON, Canada

gDepartment of Epidemiology and Biostatistics, University of Western Ontario, London, ON, Canada

${ }^{\mathrm{h}}$ Corresponding Author: William Hodge, Ivey Eye Institute, University of Western Ontario, 268 Grosvenor St, London, ON N6A 4V2, Canada

\footnotetext{
Manuscript accepted for publication January 20, 2017

avey Eye Institute, University of Western Ontario, London, ON, Canada

bFaculty of Medicine, Queen's University, Kingston, ON, Canada

'Ottawa Hospital Research Institute, University of Ottawa, Ottawa, ON, Canada

dDepartment of Ophthalmology and Visual Sciences, University of Toronto, Toronto, ON, Canada

eDepartment of Ophthalmology, University of Alberta, Edmonton, AB, Canada

fProgram for the Assessment of Technology and Health (PATH), and Department of Clinical Epidemiology and Biostatistics, McMaster University, Hamilton, ON, Canada

gDepartment of Epidemiology and Biostatistics, University of Western Ontario, London, ON, Canada

${ }^{\mathrm{h}}$ Corresponding Author: William Hodge, Ivey Eye Institute, University of Western Ontario, 268 Grosvenor St, London, ON N6A 4V2, Canada.

Email: William.hodge@sjhc.london.on.ca
}

doi: https://doi.org/10.14740/jocmr2643wc1 